\title{
Comment on "Linear confinement of a scalar particle in a Gödel-type space-time [Eur. Phys. J. C (2018) 78 : 44]”'
}

\author{
Faizuddin Ahmed ${ }^{\mathrm{a}}$ \\ Ajmal College of Arts and Science, Dhubri 783324, Assam, India
}

Received: 11 July 2019 / Accepted: 31 July 2019 / Published online: 14 August 2019

(C) The Author(s) 2019

\begin{abstract}
We point out an incorrect expression in a recent paper published in this journal (Vitória et al. Eur Phys J C, 78:44, 2018) regarding solution for the Klein-Gordon equation without potential in the background of SomRaychaudhuri space-time with the cosmic string. The obtained eigenvalue of energy there is not similar to the result in Carvalho (Eur Phys J C 74: 2935, 2014).
\end{abstract}

In a recent paper in this journal, Vitória et al. [1] have studied relativistic quantum dynamics of a scalar particle subject to a linear scalar potential in the background of SomRaychaudhuri space-time with the cosmic string. The authors obtained the bound states energy eigenvalues and the corresponding wavefunctions. The obtained expression Eqs. (5)(21) in Ref. [1] are correct. After that, they have obtained two expressions Eqs. (22)-(23) for the same metric without any potential which are seem to be incorrect. The eigenvalue of energy Eq. (23) is not similar to the result Eq. (14) obtained in Ref. [2]. In this paper, we derive the final form of the KleinGordon equation in the background of Som-Raychaudhuri space-time with the cosmic string without any potential, and solve it using the method of Nikiforov-Uvarov [3]. We then obtain the correct expression of the energy eigenvalue which is similar to the result obtained in Ref. [2].

The Som-Raychaudhuri space-time with the cosmic string is described by the following line element (with $c=$ $\hbar=1)$ :

$d s^{2}=-\left(d t+\alpha \Omega r^{2} d \phi\right)^{2}+\alpha^{2} r^{2} d \phi^{2}+d r^{2}+d z^{2}$,

where $\alpha$ and $\Omega$ respectively characterizes the cosmic string and vorticity parameter of the space-time.

Article Related Object https://doi.org/10.1140/epjc/ s10052-018-5524-7.

a e-mail: faizuddinahmed15@gmail.com
The relativistic quantum dynamics of free spinless particle of mass $M$ is described by the Klein-Gordon equation:

$\frac{1}{\sqrt{-g}} \partial_{\mu}\left(\sqrt{-g} g^{\mu \nu} \partial_{\nu} \Psi\right)=M^{2} \Psi$

with $g$ is the determinant of the metric tensor with $g^{\mu \nu}$ its inverse.

One can introduce a scalar potential into the KleinGordon equation by modifying the mass term $M$ as $M \rightarrow$ $M^{\prime}=M+S$ where, $S$ is the scalar potential. Thereby, the KG-equation (2) becomes

$\frac{1}{\sqrt{-g}} \partial_{\mu}\left(\sqrt{-g} g^{\mu \nu} \partial_{\nu} \Psi\right)=(M+S)^{2} \Psi$

The Klein-Gordon equation in the Som-Raychaudhuri space-time (1) with a linear scalar potential $\left(S(r)=k_{L} r\right)$ is given by

$$
\begin{aligned}
& -\frac{\partial^{2} \Psi}{\partial t^{2}}+\frac{1}{r} \frac{\partial}{\partial r}\left(r \frac{\partial \Psi}{\partial r}\right)+\left(\frac{1}{\alpha r} \frac{\partial}{\partial \phi}-\Omega r \frac{\partial}{\partial t}\right)^{2} \Psi+\frac{\partial^{2} \Psi}{\partial z^{2}} \\
& =\left(M+k_{L} r\right)^{2} \Psi
\end{aligned}
$$

where $k_{L}$ is the linear confining constant. One can write the solution to Eq. (4) as

$\Psi(t, r, \phi, z)=e^{i(E t+l \phi+k z)} \psi(r)$,

where $E$ is the energy of the scalar particle, $l=0, \pm 1, \pm 2$, $\pm 3, \ldots$ are the eigenvalues of the $z$-component of the angular mpmentum operator, and $k$ are the eigenvalues of the $z$-component of the linear momentum operator.

Substituting the solution Eq. (5) into the Eq. (4), one obtain

$$
\psi^{\prime \prime}(r)+\frac{1}{r} \psi^{\prime}(r)+\left(\lambda-\omega^{2} r^{2}-\frac{l^{2}}{\alpha^{2} r^{2}}-2 M k_{L} r\right) \psi(r)=0
$$


where $\omega=\sqrt{\Omega^{2} E^{2}+k_{L}^{2}}$ and $\lambda=E^{2}-M^{2}-k^{2}-\frac{2 \Omega E l}{\alpha}$. Defining a new variable $x=\sqrt{\omega} r$, then Eq. (6) becomes

$\psi^{\prime \prime}(x)+\frac{1}{x} \psi^{\prime}(x)+\left(\beta-x^{2}-\frac{l^{2}}{\alpha^{2} x^{2}}-\theta x\right) \psi(x)=0$,

where

$\theta=\frac{2 M k_{L}}{\omega^{\frac{3}{2}}}, \quad \beta=\frac{\lambda}{\omega}$.

The solution to Eq. (7) is given by

$\psi(x)=x^{\frac{|l|}{\alpha}} e^{-\frac{x^{2}}{2}} e^{-\frac{\theta}{2} x} H(x)$,

where $H(x)$ is the solution to the biconfluent Heun equation which has the form

$$
\begin{aligned}
& H^{\prime \prime}(x)+\left[\frac{1+\frac{2|l|}{\alpha}}{x}-\theta-2 x\right] H^{\prime}(x) \\
& +\left[\beta+\frac{\theta^{2}}{4}-2-\frac{2|l|}{\alpha}-\frac{\theta\left(1+\frac{2|l|}{\alpha}\right)}{2 x}\right] H(x)=0 .
\end{aligned}
$$

Substituting the power series solution $H(x)=\sum_{j=0}^{\infty} d_{j} x^{j}$ into the Eq. (10), the following recurrernce relation one obtained:

$$
\begin{gathered}
d_{j+2}=\frac{\theta\left(2 j+3+\frac{2|l|}{\alpha}\right)}{2(j+2)\left(j+2+\frac{2|l|}{\alpha}\right)} d_{j+1} \\
-\frac{\left(4 \beta+\theta^{2}-8-\frac{8|l|}{\alpha}-8 j\right)}{4(j+2)\left(j+2+\frac{2|l|}{\alpha}\right)} d_{j} .
\end{gathered}
$$

A polynomial solution to $H(x)$ is achieved by imposing the following two conditions

$4 \beta+\theta^{2}-8-\frac{8|l|}{\alpha}=8 n, d_{n+1}=0(n=1,2,3,4, \ldots)$.

The authors obtained formulae Eqs. (5)-(21) in Ref. [1] are correct. After Eq. (21), a special case correspond to $k_{L}=0$ have been addressed and obtained two expression (22)-(23) in Ref. [1] which are incorrect. The eigenvalue of energy (23) obtained in Ref. [1] is not similar to the result (14) in Ref. [2]. To obtain the same, one should start from Eq. (4) by substituting $k_{L}=0$ there but not from the above condition (12) by substituting $\theta=0$ and replacing $n \rightarrow n^{\prime}$ as done in Ref. [1] where, $n^{\prime}=0,1,2,3, \ldots$. Moreover, for $\theta=0$ the Eq. (10) does not become the hypergeometric like equation $x F^{\prime \prime}(x)+(\eta-x) F^{\prime}(x)+\gamma F(x)=0$. In this paper, we correct it and evaluate the energy eigenvalue.

Substituting $k_{L}=0$ into the Eq. (4) and using (5), we obtain the following radial wave-equation:

$\psi^{\prime \prime}(r)+\frac{1}{r} \psi^{\prime}(r)+\left(\lambda-\Omega^{2} E^{2} r^{2}-\frac{l^{2}}{\alpha^{2} r^{2}}\right) \psi(r)=0$.

The analytical solution of the above equation can be obtained by different methods. In this work, we choose the NikiforovUvarov method and obtain the eigenvalue of energy.

By doing a transformation $x=\Omega E r^{2}$ into the Eq. (13), we obtain

$\psi^{\prime \prime}(x)+\frac{1}{x} \psi^{\prime}(x)+\frac{1}{4 x}\left(\frac{\lambda}{\Omega E}-x-\frac{l^{2}}{\alpha^{2} x}\right) \psi(x)=0$.

The above Eq. (14) can be expressed as

$\psi^{\prime \prime}(x)+\frac{1}{x} \psi^{\prime}(x)+\frac{1}{x^{2}}\left(-\xi_{1} x^{2}+\xi_{2} x-\xi_{3}\right) \psi(x)=0$,

where

$\xi_{1}=\frac{1}{4}, \quad \xi_{2}=\frac{\lambda}{4 \Omega E}, \quad \xi_{3}=\frac{l^{2}}{4 \alpha^{2}}$.

Compairing Eq. (15) with (A.1) in appendix, we get

$$
\begin{aligned}
& \alpha_{1}=1, \quad \alpha_{2}=0, \quad \alpha_{3}=0, \quad \alpha_{4}=0, \quad \alpha_{5}=0, \\
& \alpha_{6}=\xi_{1}, \quad \alpha_{7}=-\xi_{2}, \quad \alpha_{8}=\xi_{3}, \quad \alpha_{9}=\xi_{1}, \\
& \alpha_{10}=1+2 \sqrt{\xi_{3}}, \quad \alpha_{11}=2 \sqrt{\xi_{1}}, \quad \alpha_{12}=\sqrt{\xi_{3}}, \\
& \alpha_{13}=-\sqrt{\xi_{1}} .
\end{aligned}
$$

The eigenvalues equation using Eqs. (16)-(17) into the Eq. (A.8) in appendix is

$$
\begin{aligned}
& (2 n+1) \sqrt{\xi_{1}}-\xi_{2}+2 \sqrt{\xi_{1} \xi_{3}}=0 \\
& \Rightarrow \frac{1}{2}(2 n+1)+\frac{|l|}{2 \alpha}=\frac{\lambda}{4 \Omega E} \\
& \Rightarrow \lambda-2 \Omega E\left(2 n+1+\frac{|l|}{\alpha}\right)=0 \\
& \Rightarrow E^{2}-2 \Omega E\left(2 n+1+\frac{l}{\alpha}+\frac{|l|}{\alpha}\right) \\
& \quad-M^{2}-k^{2}=0 .
\end{aligned}
$$

The energy eigenvalue associated with $n^{\text {th }}$ radial mode is given by 


$$
\begin{aligned}
E_{n, l}= & \left(2 n+1+\frac{l}{\alpha}+\frac{|l|}{\alpha}\right) \Omega \\
& +\sqrt{\left(2 n+1+\frac{l}{\alpha}+\frac{|l|}{\alpha}\right)^{2} \Omega^{2}+M^{2}+k^{2}}
\end{aligned}
$$

where $n=0,1,2,3,4, \ldots$. The above eigenvalue of energy is similar to the result obtained in Ref. [2] (see Eq. (14) in Ref. [2]).

For $\alpha=1$, the energy eigenvalue reduces to the result obtained in Refs. [4,5]. For $M=0=k$, the energy eigenvalue (19) becomes $E_{n, l}=2 \Omega\left(2 n+1+\frac{l}{\alpha}+\frac{|l|}{\alpha}\right)$. This is similar to the energy eigenvalue obtained for the Landau levels in the presence of cosmic string in Ref. [6].

The radial wave-function is given by

$\psi_{n, l}(x)=x^{\frac{|l|}{2 \alpha}} e^{-\frac{x}{2}} L_{n}^{\left(\frac{|l|}{\alpha}\right)}(x)$

where $L_{n}^{\left(\frac{|l|}{\alpha}\right)}(x)$ denotes the generalized Laguerre polynomial. One can obtain the individual energy level and corresponding wave-function one by one substituting $n=$ $0,1,2,3,4, \ldots$.

In summary, we have solved the Klein-Gordon equation without interactions in the Som-Raychaudhuri space-time with the cosmic string using the Nikiforov-Uvarov method. We then have obtained the energy eigenvalue (19) and the corresponding eigenfunctions (20) of the system. We have seen that our eigenvalue of energy (19) is similar to the result obtained in Ref. [2] (see Eq. (14) in Ref. [2]) but different from the result in Ref. [1] (see Eq. (23) in Ref. [1]). This fact indicates that the eigenvalue of energy obtained in Ref. [1] is incorrect.

Acknowledgements The author acknowledge valuable comments and suggestions from the anonymous kind referee(s).

Data Availability Statement This manuscript has no associated data or the data will not be deposited. [Authors' comment: There is no data associated with this manuscript or no data has been used to prepare it.]

Open Access This article is distributed under the terms of the Creative Commons Attribution 4.0 International License (http://creativecomm ons.org/licenses/by/4.0/), which permits unrestricted use, distribution, and reproduction in any medium, provided you give appropriate credit to the original author(s) and the source, provide a link to the Creative Commons license, and indicate if changes were made.

Funded by SCOAP ${ }^{3}$.

\section{Appendix: Brief review of the Nikiforov-Uvarov (NU) method}

The Nikiforov-Uvarov method is helpful in order to find eigenvalues and eigenfunctions of the Schrödinger like equation, as well as other second-order differential equations of physical interest. According to this method, the eigenfunctions of a second-order differential equation $[3,7,8]$ $\frac{d^{2} \psi(s)}{d s^{2}}+\frac{\left(\alpha_{1}-\alpha_{2} s\right)}{s\left(1-\alpha_{3} s\right)} \frac{d \psi(s)}{d s}+\frac{\left(-\xi_{1} s^{2}+\xi_{2} s-\xi_{3}\right)}{s^{2}\left(1-\alpha_{3} s\right)^{2}} \psi(s)=0$

are given by

$\psi(s)=s^{\alpha_{12}}\left(1-\alpha_{3} s\right)^{-\alpha_{12}-\frac{\alpha_{13}}{\alpha_{3}}} P_{n}^{\left(\alpha_{10}-1, \frac{\alpha_{11}}{\alpha_{3}}-\alpha_{10}-1\right)}\left(1-2 \alpha_{3} s\right)$.

And that the energy eigenvalues equation

$$
\begin{aligned}
& \alpha_{2} n-(2 n+1) \alpha_{5}+(2 n+1)\left(\sqrt{\alpha_{9}}+\alpha_{3} \sqrt{\alpha_{8}}\right) \\
& +n(n-1) \alpha_{3}+\alpha_{7}+2 \alpha_{3} \alpha_{8}+2 \sqrt{\alpha_{8} \alpha_{9}}=0 .
\end{aligned}
$$

The parameters $\alpha_{4}, \ldots, \alpha_{13}$ are obatined from the six parameters $\alpha_{1}, \ldots, \alpha_{3}$ and $\xi_{1}, \ldots, \xi_{3}$ as follows:

$$
\begin{aligned}
& \alpha_{4}=\frac{1}{2}\left(1-\alpha_{1}\right) \quad, \quad \alpha_{5}=\frac{1}{2}\left(\alpha_{2}-2 \alpha_{3}\right), \\
& \alpha_{6}=\alpha_{5}^{2}+\xi_{1} \quad, \quad \alpha_{7}=2 \alpha_{4} \alpha_{5}-\xi_{2}, \\
& \alpha_{8}=\alpha_{4}^{2}+\xi_{3} \quad, \quad \alpha_{9}=\alpha_{6}+\alpha_{3} \alpha_{7}+\alpha_{3}^{2} \alpha_{8}, \\
& \alpha_{10}=\alpha_{1}+2 \alpha_{4}+2 \sqrt{\alpha_{8}}, \\
& \alpha_{11}=\alpha_{2}-2 \alpha_{5}+2\left(\sqrt{\alpha_{9}}+\alpha_{3} \sqrt{\alpha_{8}}\right), \\
& \alpha_{12}=\alpha_{4}+\sqrt{\alpha_{8}}, \alpha_{13}=\alpha_{5}-\left(\sqrt{\alpha_{9}}+\alpha_{3} \sqrt{\alpha_{8}}\right) .
\end{aligned}
$$

A special case where $\alpha_{3}=0$, we find

$$
\lim _{\alpha_{3} \rightarrow 0} P_{n}^{\left(\alpha_{10}-1, \frac{\alpha_{11}}{\alpha_{3}}-\alpha_{10}-1\right)}\left(1-2 \alpha_{3} s\right)=L_{n}^{\alpha_{10}-1}\left(\alpha_{11} s\right),
$$

and

$$
\lim _{\alpha_{3} \rightarrow 0}\left(1-\alpha_{3} s\right)^{-\alpha_{12}-\frac{\alpha_{13}}{\alpha_{3}}}=e^{\alpha_{13} s} .
$$

Therefore the wave-function from (A.2) becomes

$\psi(s)=s^{\alpha_{12}} e^{\alpha_{13} s} L_{n}^{\alpha_{10}-1}\left(\alpha_{11} s\right)$,

where $L_{n}^{(\beta)}(x)$ denotes the generalized Laguerre polynomial.

The energy eigenvalues equation reduces to

$n \alpha_{2}-(2 n+1) \alpha_{5}+(2 n+1) \sqrt{\alpha_{9}}+\alpha_{7}+2 \sqrt{\alpha_{8} \alpha_{9}}=0$.

\section{References}

1. R.L.L. Vitoria, C. Furtado, K. Bakke, Eur. Phys. J. C 78, 44 (2018)

2. J. Carvalho, A.M.de M. Carvalho, C. Furtado, Eur. Phys. J. C 74, 2935 (2014)

3. A.F. Nikiforov, V.B. Uvarov, Special functions of mathematical physics (Birkhauser, Basel, 1988)

4. N. Drukker, B. Fiol, J. Simon, JCAP 0410, 012 (2004) 
5. S. Das, J. Gegenberg, Gen. Relat. Gravit. 40, 2115 (2008)

6. C. Furtado, B.G.C. da Cunha, F. Moraes, E.R.B. de Mello, V.B. Bezzera, Phys. Lett. A 195, 90 (1994)
7. F. Ahmed, Ann. Phys. (N. Y.) 404, 1 (2019)

8. F. Ahmed, Gen. Relat. Gravit. 51, 69 (2019) 